\title{
Minimally invasive transforaminal lumbosacral interbody
} fusion

\author{
Peng-Yuan Chang, MD, and Michael Y. Wang, MD \\ Departments of Neurosurgery and Rehabilitation Medicine, University of Miami Miller School of Medicine, Miami, Florida \\ In minimally invasive spinal fusion surgery, transforaminal lumbar (sacral) interbody fusion (TLIF) is one of the most \\ common procedures that provides both anterior and posterior column support without retraction or violation to the neural \\ structure. Direct and indirect decompression can be done through this single approach. Preoperative plain radiographs \\ and MR scan should be carefully evaluated. This video demonstrates a standard approach for how to perform a mini- \\ mally invasive transforaminal lumbosacral interbody fusion.
}

The video can be found here: https://youtu.be/bhEeafKJ370.

KEYWORDS minimally invasive surgery; MIS; TLIF; expandable cage; percutaneous screw; video 